\title{
Analysis of Light Art Application in the Gallery Exhibition
}

\author{
Wei $\mathrm{Tu}^{1, *}$ Yue Jiang ${ }^{1}$ \\ ${ }^{1}$ Wuhan University of Science and Technology, Wuhan, Hubei, China \\ *Corresponding author. Email: 1007710399@qq.com
}

\begin{abstract}
Most of the light art designs in modern exhibition galleries today have two distinct characteristics: satisfying functionality and conforming to artistry. When the above two characteristics are satisfied in choosing a light source, it is necessary to meet the functions of relatively weak ultraviolet rays, excellent color rendering function, energy saving and environmental protection, and high light efficiency. While the good effect of light art in the exhibition hall is satisfied, it is also necessary to consider the unity of light perception and the environment in the exhibition hall, the satisfaction of material standards, and the budget of the expenditure. It is often hoped that lighting in art museums can change the traditional lighting style in the past to show different light effects, different art styles and the unique charm of exhibition halls.
\end{abstract}

Keywords: light art, exhibition lighting, art gallery lighting, lighting design

\section{INTRODUCTION}

According to surveys, the problems that need to be avoided at present are mainly: first, the color temperature is too high or too low, which leads to deviations in the display effect of the work; second, the insufficient color rendering of the light illumination, which causes the work to not fully display its own effects; third: insufficient or uneven light intensity leads to too large areas of light spots or dark sides of objects, which affects the viewing effect of works and viewers; fourth: at any time, a single lighting method cannot make light art add artistic charm or help to the work, making it useless.

\section{THE ROLE OF LIGHT ART IN THE GALLERY}

\section{A. Color temperature}

Color temperature is a physical quantity that defines the color of a light source. It is also a light metric. The physical explanation is a general indicator of the spectral quality of the light source, and its unit is $\mathrm{k}$. In the research, it is found that in light sources with low color temperature, there are fewer cold light components and more warm light components; on the other hand, in light sources with high color temperature, cold light is more and warm light is less (cold light is a blue series light source, and warm light is a red light series light source). In the exhibition hall, the color temperature is sought to the greatest extent. It is not only the requirements of the works, but also the requirements of the viewers and the space itself. In the exhibition hall, the color temperature is to seek the highest degree of unity, not only for the requirements of the works, but also for the requirements of the viewers and the space itself.

After investigation, it is found that visitors' viewing and shopping desires in the light source with low color temperature are relatively easy to be impulsive, and their emotions are also prone to instability. Although there is a moment of pleasure, it is uncomfortable to stay in this space for a long time, as shown in "Fig. 1". On the contrary, in a light source with a high color temperature, the atmosphere may not be as good as a light source with a low color temperature. But for visitors, it makes people relatively calm in thought, easier to think, and staying in this space for a long time without being repressed. The application of color temperature in the exhibition hall of the art museum is only a way of using light art in essence. Its main purpose is to show the work itself and a shaping of the spatial vision. (See "Fig. 2")

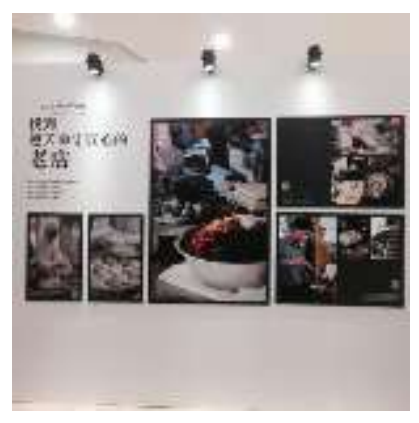

Fig. 1. Example of low color temperature. 


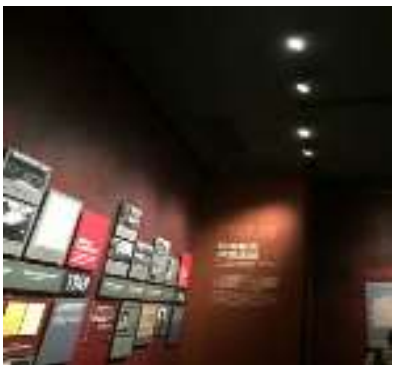

Fig. 2. Example of high color temperature.

\section{B. Color rendering}

Color rendering refers to the characteristics of different hue colors displayed by light sources with different spectra on the same object. At present, the color rendering of a light source is physically summarized as the color rendering index, represented by (ra). The so-called high color rendering index is that what reappears in front of visitors' eyes is complete by the effect of the light source on the object's own tonal color. For the color rendering of light art in the gallery of the art museum, it will be a must to consider the material of the object being displayed and the characteristics of the material itself, so that the color rendering is even higher when it is arranged.

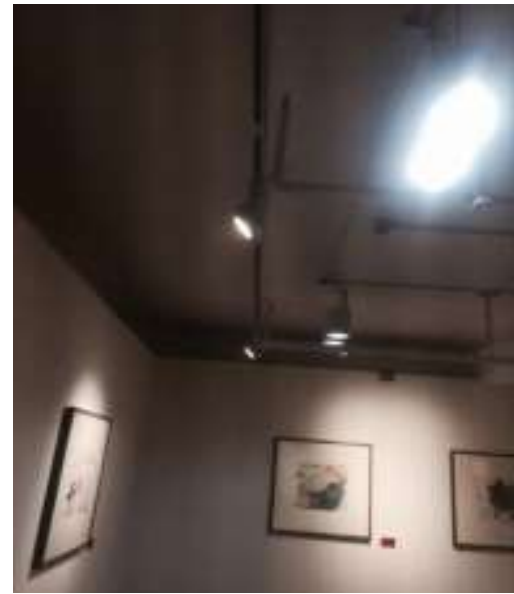

Fig. 3. Example of high color rendering.

In terms of color rendering, the completeness of the natural color of the object shown in "Fig. 3" is a fundamental problem. After removing ultraviolet rays and some red light, the issue of color rendering can be considered to the greatest extent in the exhibition hall, and they must adapt to each other and consider each other. When reflecting the natural colors of the objects in the exhibition hall, it must also consider the changes caused by the reflection or refraction of the light source hanging from the surrounding walls.

In the exhibition halls of art museums, the difference in color rendering can be used to divide the space, shape the vision in the space, and better use light art in the space.

\section{Illumination}

Illumination refers to the amount of light received by the "light intensity" per unit area of an object, which is a physical interpretation. When considering the illumination, it is necessary to pay attention to the area size of the light receiving surface, the projection surface and the dark surface so that they do not influence each other and cause flare problems or glare problems that affect the effect of the work.

According to the research, it is found that the "dim art style" is popular in modern art museums and some related exhibition halls.

In fact, from the perspective of science and visitors, this is counterproductive, and it is easier for visitors to have visual fatigue and inconvenience in appreciating the works. In another way, the intensity of the illumination is the brightness of the light. The stronger the illumination, the brighter the light is, and the weaker the illumination, the darker the light is. People have an innate phototaxis response to light. In the gallery exhibition hall, the intensity of illumination can be used to divide the space and guide the visitors' paths, so as to achieve the function of dividing space and crowd flow. On the issue of illumination, it is divided into natural light and artificial light. The intensity of natural light is closely related to the sunrise and sunset. The artificial light's illumination is widely used in the exhibition halls of art museums, but it is too simple in terms of the number and the varieties and does not really reflect the meaning of its illumination. In terms of illumination, it can be used to distinguish between space and light to shape the spatial intention, and it can also be used as a guide in space. (See "Fig. 4" and "Fig. $5 ")$

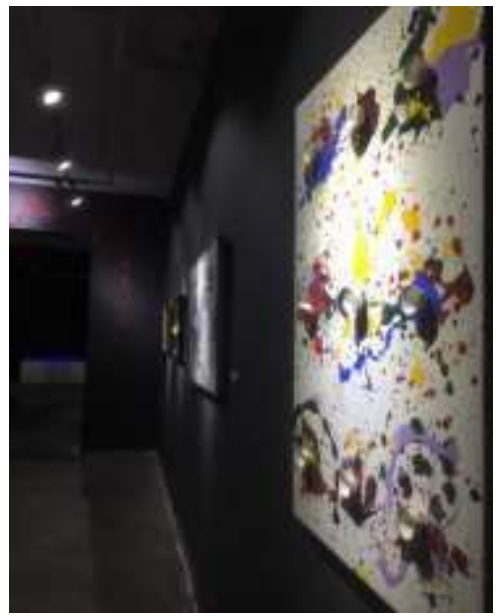

Fig. 4. Example of weak light 


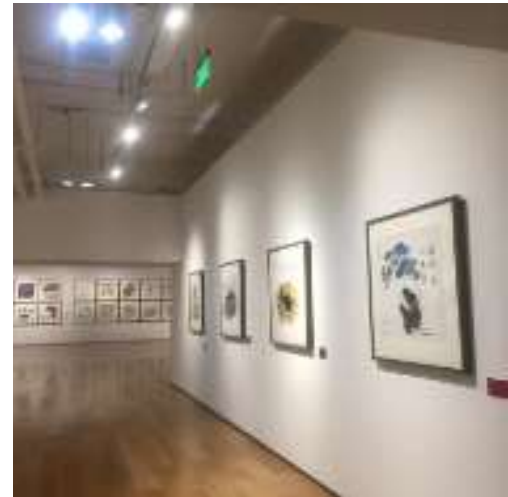

Fig. 5. Example of strong light

\section{THE INFLUENCE OF LIGHT ART ON SPACE IN THE ART GALLERY}

\section{A. Impacts on the work}

There is a change in human perception of an object, and this change is affected by many factors. The factors affected the work are caused by the joint influence of light art and color factors of the work. From this, it is known that both in the works and light arts are diverse.

According to the investigation and research, it is found that in many cases, the light in the museum's exhibition hall can only be called lighting but not light art. Most of the time, it is directly applied to the existing template or related series of standards and is not designed. There are even some designers using subjective imagination to arbitrarily design without considering the actual situation and the materials and characteristics of the work itself. Due to various problems, the natural colors that should be reflected in the work itself are not reflected. Originally, the works in the gallery's exhibition hall are to remind the designers of their own ideas and opinions. It is a pity that they cannot be reflected due to the deviation of external conditions.

The above are just some of the problems discovered during the survey. The scientific basis and artistic design are not harmoniously integrated, and naturally it cannot reflect the work itself and the light art to the greatest extent, and it cannot be optimized. All in all, this is certain blindness, and it is not clear. It needs to be artistically optimized on the basis of science.

\section{B. Impacts on visitors}

1) Psychological impact: In the gallery exhibition hall, the light art is a kind of aggregation effect created between the Western painting tools and Chinese consciousness. It is a very charming art form. This light art is to give people a sense and feel outside of the work. Good light art can add artistic charm to the work.
Otherwise, it will make the viewer have a kind of fatigue. The influence of light art on visitors' psychology in color temperature, illumination, and color rendering is mainly reflected in as follows:

- When the color temperature is high, the visitors will have a moment of pleasure. But if staying in such an environment for a long time, the visitors' emotions will be prone to instability. In contrast, when the color temperature is cold, the viewer's mood will be more calm and easy to think. Then, when the color rendering is high, the visitors can intuitively feel the color or emotion of the work; on the contrary, when the color rendering is low, the color of the work will be deteriorated and distorted, so that the visitors cannot directly feel the charm of the work. Finally, when the illumination is high, the visitors can intuitively appreciate the work, and the recent "dim style" is not suitable for the visitors to appreciate the work.

- A clever use of light art in the gallery's exhibition hall will greatly assist the visitors in their shopping desire or the degree of appreciating the works. A good light art atmosphere can make the visitors in a wonderful situation, which is essential on the work and the deeper understanding of the work.

- Light art is changeable. Human emotions are also changeable, and it is due to multiple factors. Therefore, the use of light art in the exhibition halls of art museums should be based on the sensory feelings of people when considering the display of works to reduce the adverse effects of light art on people.

2) Physiological impact: In the gallery's exhibition hall, the light art's processing method has caused some inhumane problems due to the subjective arbitrariness of its designers or the blind pursuit of science. The impact of the use of light art on the physiological aspects of visitors is mainly reflected in the following:

- Insufficient or uneven illumination causes flare or glare problems, which causes visitors' discomfort in the eyes or cerebral cortex when viewing (that is, the moment people close the eyes, they feel starlight flickering or black dots bouncing in front of their eyes).

- The insufficient color rendering of lighting in light art can easily lead to the deterioration of the color of the work, making the visitors unable to correctly feel the charm of the work, and the visitors will be prone to visual fatigue when watching.

- Due to some lighting angles or reflection problems, glare will occur, that is, in a relatively 
dim environment for a long time, when suddenly being illuminated by a strong light source, the eyes cannot accept the stimulus of strong light and produce a strong discomfort, which can cause tears and even irreversible damage to the eyes.

In the exhibition hall of the art museum, a reasonable and scientific use of light art must be comfortable and enjoyable.

\section{OPTIMIZATION SUGGESTIONS AND DEVELOPMENT TRENDS OF THE USE OF LIGHT ART IN THE EXHIBITION HALLS OF ART MUSEUMS}

\section{A. Problems to be avoided and ways to overcome them}

According to surveys, the problems that need to be avoided at present are mainly: the first is that the color temperature is too high or too low, which leads to deviations in the display effect of the work; the second is the insufficient color rendering of the light illumination, which causes the work to not fully display its own effects; the third is that insufficient or uneven light intensity leads to too large areas of light spots or dark sides of objects, which affects the viewing effect of works and viewers; the forth is that at any time, a single lighting method cannot make light art add artistic charm or help to the work, making it useless.

In China, both economy and science are developing rapidly. Now, unlike the original closed-door and selfstyled country, China is learning from the advanced practices of some advanced countries in the West. It is necessary to proceed from the facts. According to the actual situation and combined with it, through the indepth exploration of the problem, the following methods are summarized to overcome the current major problems:

- From the work itself, whether it is the choice of artificial lighting or the preservation of natural light, it should consider and control the ultraviolet content. And when optimizing the rectification, it is a must to pay attention to the preservation and use of natural light, and consider using reflection, refraction and other methods to form diffuse reflection or scattering to achieve the optimization effect.

- When using artificial lighting, it is necessary to consider the relationship between the intensity of illumination and the distance between the light and the texture, material, and expression of the work. (For example, when the illumination of oil painting works is $150 \mathrm{~lx}$ color temperature $\leq 4000 \mathrm{~K}$, the illumination of Chinese painting and ink painting is $50 \mathrm{~lx}$ color temperature $\leq$ 2900K.)

- When artificial lighting is used, the occurrence of glare, light spots and moiré should be considered. When setting the lighting system, the angles, materials and illumination should be adjusted to a suitable level to avoid the above problems.

1) Sustainable development: Sustainable development is a big trend in today's society and one of the basic principles of China. It is a must to consider the issues of the combination of the use of light art in the exhibition halls of art museums and sustainable development. According to the research, it can be seen that a light art sustainable development in the exhibition hall of the art museum must be dynamic and must have relatively rich functional areas to meet different needs. The following aspects should be achieved to meet sustainable development:

- First of all, it will be necessary to divide the overall illumination, color temperature and color rendering of the lighting in the exhibition hall, and highlight the key points in the exhibition hall to reflect the levels in the works in order to be able to play the role in the true sense. It will also be necessary to consider the extent to the usage of natural light when the seasons change.

- Secondly, historical culture, natural aesthetics should be sustainable, the lighting method should be scientific, efficient, low-consumption sustainable, and the lighting system should be effective and sustainable.

- Finally, it is necessary to avoid blindly pursuing illuminance, the disorderly use of a variety of lamps, and the messy layout. It is strictly forbidden to use unprincipled lighting that does not help the works in the exhibition halls of the art museum. It is a must to be against with the abuse of LED products, and more consideration should be given to parameterized and quality lighting.

2) Energy saving and emission reduction: Light is a magical existence that people can see but can't touch, and it is indispensable. Daylighting is indispensable in the energy-saving and low-carbon economy. Full use of natural light as a lighting source is of great significance in the design. The current situation of energy saving and emission reduction is grim. The government gives strong support in this regard. As a new type of energy saving industry, LED is well received by the public and supported by the government. The energy saving and emission reduction issues in the consideration are based on sustainable development. The former is to 
fundamentally consider the problem, and the latter is to fundamentally solve the problem. According to the investigation, the light guide lighting system can transmit natural light to places where the light couldn't reach, so that the problems of lighting on many buildings can be solved and improved. It can be used for the lighting in the exhibition hall of the art museum. It can be built on the basis of not affecting the existing structure in the exhibition hall of the art museum. Not only does it have no energy consumption, dust-proof, waterproof, sound insulation, but also can further improve the quality of the indoor environment, save a lot of energy, and at the same time create a comfortable environment in the exhibition hall, thereby achieving the purpose the researchers have thought.

The combination of the use of LED lighting, energy saving, emission reduction, and energy saving transformation, is technically capable of replacing traditional light sources (such as color temperature, color rendering, illuminance, life and reliability, etc.), and is economically cost-effective. The cost of LED is a major disadvantage. However, with the strong support of the government, the status quo has been improved, thereby further highlighting the advantages. According to the research, it has been learned that many countries in the world are paying more and more attention to energy conservation and environmental protection. Many countries have introduced a large number of policies to encourage energy conservation and emission reduction, and limit the application of products with high energy consumption. The production and sales of bulbs under 15 watts were cancelled. In August 2015, China first implemented government procurement compensation and subsidies on costs for LED products. From minority use to widespread recognition, the market prospect of LED lighting has spread all over the streets.

In the future, from the perspective of energy conservation and emission reduction, the use of light art in the exhibition halls of the art gallery will be combined with daylighting and LED lighting to reflect the artistry in science and the scientific nature of light art Design.

\section{B. Future development trend of light art in the galleries of art museums}

Light is now detached from its original meaning and transformed into a spiritual idea and a symbol in meaning. In the author's opinion, the main reason why light art is called contemporary "visual" art in today's society is that it uses "light" as a medium and can simply and directly reflect the connectivity, permeability and convertibility between an object itself and perception. Light can exists as a plane, a space, and any possible existence. The author thinks the art of light in the museum's exhibition hall should focus on the present and serve the future - being people-oriented and sustainable development.

The first priority of any light art design or architecture or everything else is to serve people. However, at the same time, it must show respect for itself, consider the corresponding environment in the museum's own exhibition hall, integrate the light art with the environment in the exhibition hall, and adapt the light art to the relevant environment. This is one of the most important parts. Secondly, it is a must to consider that light art must meet the functional needs of users.

The specific issues considered are not only the application of light art, but also the comprehensive consideration. The first is to satisfy its functionality and its uniqueness on the basis of it. In the past, the form of unilaterally relying on artificial lighting in the museum's exhibition halls will change. Pure artificial lighting cannot meet the lighting requirements in the museum's exhibition halls. The design of light art in the exhibition halls of the future will combine artificial lighting and natural light lighting. Due to the continuous development of modern technology and the continuous improvement of aesthetic requirements, this has also provided a better market environment for the development of light art in the exhibition halls of future art museums. At the same time, people's awareness of design is constantly increasing and their demands are increasing. Facing it seriously, innovation and development is a very important attitude. The light art in the exhibition hall of the art museum should create a comfortable environment space that meets people's needs. Nowadays, it can't just be satisfied with the beauty of creation forms. Scientific artistry and being able to continue to develop such design concepts will become an important trend for the future development of light art in the museum's exhibition halls.

\section{CONCLUSION}

In China, both economics and science are developing rapidly. Now, unlike the original closeddoor country, which is closed-door and self-styled, it will be of great significance to learn from the advanced practices of some advanced countries in the West. It will be necessary to start from the facts and combine it with Chinese actual situation.

\section{References}

[1] Ding Zongjiang, Art Gallery Lighting and Display of Works of Art (China Art Museum, Beijing 100010).

[2] Zheng Ying, etc. Application of LED Bionic Lighting in Museum Lighting. (in Chinese) 
[3] Jiang Yuan, Light Source for Polymer Optical Fiber Beam Transmission and Application (Vol. 1), Nanjing Fiberglass Research \& Design Inistitute. (in Chinese)

[4] Li Hui, iGuzzini: The Wonderful Connection of Light and Art.

[5] National Cultural Heritage Administration, Notice of the National Cultural Heritage Administration on Issuing the "Specifications for the Design of Museum Lighting". (in Chinese)

[6] Zhang Xin, Zhan Qingxuan, History and Present of the Luminous Environment in Domestic Museums - Taking the National Art Gallery as Example [J]. Architectural Journal. (in Chinese)

[7] National Technical Committee for the Protection of Cultural Heritage. GB/T 23863-2009 Museum Lighting Design Code [S]. Beijing: Standards Press of China, 2009. (in Chinese)

[8] Dang Rui, Zhang Mingyu, Liu Gang, etc. Investigation and Research on Display Lighting in Museum Based on Protected Historical Relics [J]. China Illuminating Engineering Journal, 2013, 24 (3): 18-23. (in Chinese)

[9] Su Xiaomeng, Status Quo Analysis and Improvement Research on Lighting Designs for Museums [J]. China Light \& Lighting, 2009(8): 7-11. (in Chinese)

[10] Zhou Linglin, Research on Lighting Design of Modern Museum - Based on the Investigation of Anhui Museum [J]. Journal of Zhengzhou University of Light Industry (Social Science), 2015, 16. (in Chinese)

[11] Zhang Huan, Discussion on Museum Illumination Based on Conservation of Culture Heritage $[\mathrm{J}]$. China Illuminating Engineering Journal. (in Chinese)

[12] Chen Yuansheng, Xie Yulin, Studies on Museum Environmental Standards [J]. Sciences of Conservation and Archaeology, 2002, 14(Supplement): 152-191. (in Chinese)

[13] National Technical Committee for the Protection of Cultural Heritage. GB/T 23863-2009 Museum Lighting Design Code [S]. Standards Press of China. (in Chinese)

[14] Zhang Huan, Discussion on Museum Illumination Based on Conservation of Culture Heritage [J]. China Illuminating Engineering Journal, 2012(6): 64-67. (in Chinese)

[15] Liu Muqing, Far Influence of LED on Lighting Science [J]. China Illuminating Engineering Journal, 2014, 25(1): 15-20. (in Chinese)

[16] Sun Chengqun, Museum Lighting Design [J]. Intelligent Building and Smart City, 2004 (8): 22 -24. (in Chinese)

[17] Zhu Hong, Research of the Factors that Affect Lighting Environment of Modern Exhibiting Hall [J]. China Illuminating Engineering Journal, 2005, 16(3). (in Chinese)

[18] Chen Dahua, Lighting for Museums and Galleries [Z]. Institute of Electric Light Sources, Fudan University. (in Chinese)

[19] Fan Tongshun, Su Wei, Lighting Project Design of Art Museum [D]. Beijing Union University. (in Chinese)

[20] Chen Jiao, Daylighting and Lighting of Exhibition Building [J]. China Illuminating Engineering Journal, 2014, 25(1): 15-20. (in Chinese)

[21] Lin Fangyi, "Creating a Friendly Urban Cultural Place" [J]. Taibei: Yanhuang Art, 1994, 3. in Chinese) 